\title{
Apparent movement and metacontrast: A note on Kahneman's formulation
}

\author{
NAOMI WEISSTEIN ${ }^{2}$ AND RONALD L. GROWNEY \\ LOYOLA UNIVERSITY, CHICAGO
}

\begin{abstract}
Recently metacontrast has been described as a case of "impossible" apparent motion. Kahneman (1967) has supported this hypothesis with a study showing that apparent motion and metacontrast functions resemble one another. However, when visual angle, luminance, and duration vary from the values used in the Kahneman study, the resemblance between the two effects breaks down. Since the apparent motion explanation for metacontrast assumes an identity between apparent motion and metacontrast, these present results indicate that the apparent motion hypothesis for metacontrast is untenable. Metacontrast and apparent motion may not be unrelated, however; they might both have some thing to do with single units in the visual cortex. For each effect, however, different functional units would be involved.
\end{abstract}

Among visual masking effects, metacontrast and paracontrast have seemed most elusive and difficult to explain (Schiller, 1968; Kahneman, 1968; Eriksen, Collins, \& Greenspon, 1967). Most of the time in visual masking, the extent to which a target stimulus is suppressed is a decreasing monotonic function of the delay between the onset of the target stimulus and the onset of the masking stimulus $(\Delta t)$. The major feature of metacontrast and paracontrast is that for a broad class of stimulus paradigms-those in which the target and mask energies are roughly equal-the target stimulus is maximally suppressed at nonzero values of $\Delta t$, yielding the so-called "Y-shaped" masking function. (On the other hand, for another broad class of metacontrast and paracontrast paradigms-when the mask is of greater energy than the target-decreasing monotonic masking functions, similar to other types of visual masking functions, are obtained.)

Metacontrast and paracontrast can be defined by the spatial relations of the target and the mask; the mask is larger than the target, but it is in no way superimposed on the target. Typical targets have been discs with annuli as masks, or central squares which are flanked by two adjacent masking squares. Dichoptic presentations are as, if not more, effective than monoptic presentations.

Most general theories of visual masking assume that, in some sense, information about the masking stimulus gets relayed ahead of, or at the same time as, the information about the target stimulus and, thus, "blocks" or substitutes for the target stimulus. This is assumed to happen for one of several reasons: because the masking stimulus is of higher energy and, therefore, of shorter latency than the target stimulus and, hence, either causes adaptation such that the target stimulus is below threshold or causes asymptotic firing such that the target signal is lost (Schiller, 1968); or because the target and mask summate in such a way as to simultaneously suppress information that there were two flashes, rather than one, present (Landahl, 1968); or because the mask laterally inhibits the target stimulus (Ratliff, 1963; Dember \& Purcell, 1967; Weisstein, 1968, 1969). Since metacontrast and paracontrast involve adjacent, not overlapping, stimuli and are found with target-mask arrangements similar to those for single unit receptive fields, lateral inhibition seems a promising candidate for an explanation of these effects. But lateral inhibition has generally been rejected on the basis that it would not yield
U-shaped functions; if two stimuli are presented simultaneously, they they should be inhibited simultaneously. Implicit in this rejection, however, is the notion that hyperpolarization and depolarization slow potentials must develop at the same rates, for Weisstein (1968) has shown that if it is assumed that a hyperpolarization slow potential develops at a faster rate, then metacontrast masking maxima will occur at nonzero $\Delta t$ 's for approximately equal energy stimuli, and at zero $\Delta$ t's for stimuli where the mask is of much greater energy than the target. (For paracontrast, it would be assumed that a hyperpolarization slow potential develops at a slower rate.) This assumption, then, will account for both U-shaped and monotonic metacontrast functions by a single mechanism.

An alternate explanation, which assumes that U-shaped functions are mediated by one type of mechanism and monotonic functions are mediated by another, sees the former as an example of apparent movement and the latter as an example of visual masking (Kahneman, 1967; Schiller \& Smith, 1966; Fehrer, 1966). The target is occluded for U-shaped functions, according to this view, because it moves into the mask, thus creating the perceptual confusion that it was not there at all. Generally (Kahneman, 1967), monotonic functions are then assumed to be mediated by lateral inhibition.

This paper argues that the apparent movement explanation in general and Kahneman's elaboration of it in particular, is neither parsimonious nor adequate to handle the empirical evidence. It shows that under conditions where good metacontrast functions can be obtained, the functions for apparent movement and metacontrast are not the same, and they obey different laws. The paper concludes by noting that the effects are not unrelated; they cannot explain one another, but they may both have something to do with the activity of single units in the visual cortex.

\section{APPARENT MOVEMENT: GENERAL CRITIQUE}

Apparent movement typically yields a U-shaped function of the delay between the first and second stimulus. Kahneman (1967) hypothesized that the metacontrast paradigm provides a case where the motion perceived is physically impossible; the target is seen simultaneously moving towards two squares which are on either side of it or it is seen "expanding" into its annulus. Since these perceptions do not make physical sense, they are modified; the appearance of the target stimulus is degraded or entirely suppressed. As noted, this explanation is assumed to apply only to equal energy targets and masks, since unequal energy targets and masks yield monotonic functions, whereas, apparent movement would still yield U-shapes for these latter stimuli. Metacontrast in this latter case is then assumed to be due to lateral inhibition.

There are a number of theoretical difficulties with this formulation. First, it is not clear under this hypothesis why one would obtain forward masking, or paracontrast effects. If impossible motion produces masking, there are two possibilities when the mask is presented first and the target second; both of these possibilities predict that the target will be seen and the mask occluded. Either the presentation is not impossible motion (such as the case when two squares move 
towards a center square), in which case both the target and the masks should be seen, or the presentation is impossible motion (such as perhaps is the case when an annulus "shrinks" into a disc), in which case the mask should disappear and the target should be seen. Either way, the apparent movement hypothesis predicts that no paracontrast effect would be obtained.

The designation of what is and what is not impossible motion is further confused by the finding that a target will reappear under conditions in which both the target and the mask are repeatedly presented with delays between one target-mask cycle and another with equal delays between the target and mask. With a disc and annulus, the target reappears with this recycling (Schiller \& Smith, 1966), yet the movement of the target into the mask is "paradoxical" on every cycle.

Although a theory does not stand or fall on its parsimony, there is a serious ad hoc quality to Kahneman's formulation. The levels of analysis for the variety of metacontrast and paracontrast effects differ depending on the energy ratios of the target and mask, while all other stimulus conditions are identical. Unequal energies are assumed to produce an effect due to lateral inhibition, a neurophysiological mechanism; equal energies are assumed to produce an effect which can only be understood in terms of the "grammar" of perception. This grammar operates on a higher level of perceptual functioning where physical impossibilities in the environment are corrected. This shift in levels, however, simply elaborates the problem; now apparent movement has to be explained on the neurophysiological level, and both apparent movement and metacontrast have to be explained in addition on the level of perceptual grammar.

\section{APPARENT MOVEMENT AND METACONTRAST:} PREDICTED DIFFERENCES

Kahneman's hypothesis says, in effect, that for equal energy stimuli, where "impossible motion" can be assumed, metacontrast is apparent motion. Thus, his hypothesis is supported under equal energy conditions of impossible motion whenever metacontrast and apparent motion yield the same function.

He reported a study (1967) using lighted outline squares: an adjacent square, a center square, and another adjacent square were presented successively to obtain an apparent motion effect; and a center square and two adjacent squares were presented successively to obtain a metacontrast effect. He had naive Ss rate the "goodness" of both effects on a category rating scale from 0 to 5 , with large ratings indicating good motion in the apparent motion condition, and with large ratings indicating effective target suppression in the metacontrast condition. He used five durations of target and mask: $25,50,75,100$, and $125 \mathrm{msec}$, with target duration always equal to mask duration. All other parameters, luminance, visual angle, size of squares, were kept constant. He found that the functions describing apparent motion for all five durations did not appear to differ from the functions describing metacontrast. No statistical tests between metacontrast and apparent motion functions were reported in his study.

The results of Kahneman's study support his hypothesis, since apparently metacontrast and apparent motion do not differ from each other. Now, conversely, if it can be shown for equal energy stimuli where impossible motion can be assumed (three squares or a disc and annulus) that metacontrast and apparent motion don't generally yield the same function, then the hypothesis is contradicted. Thus, if parametric studies comparing apparent motion and metacontrast (with equal energy stimuli, and "impossible motion" conditions) yield different functions of $\Delta t$, it can be concluded that metacontrast is not apparent motion.
The parameters on which apparent motion and metacontrast would be expected to differ depend jointly on what is known empirically about the two types of effects and on the particular models for each effect. We had a specific lateral inhibition model for metacontrast (Weisstein, 1968, 1969), and we had a tentative, qualitative model for apparent motion. Both models assumed that the two effects were mediated by specific, different neural circuits. Metacontrast was assumed to reflect the antagonistic action of a collection of closely overlapping receptive fields, with the mask and the target together comprising the diameter of the field and the two stimuli falling on antagonistic regions of the field. Apparent motion $^{3}$ was assumed due to the firing of a collection of "motion-sensitive" single units, such as those described by Barlow, Hill, and Levick (1964) and Barlow and Levick (1965) for the rabbit's retina. These units simply sum firing from successive stimulation of the center of the receptive field, with stationary points producing only brief initial bursts, and unilateral inhibition suppressing successive stimulation in one direction and not the other.

Certain predictions follow from these models. First, consider the assumption that metacontrast is mediated by receptive field antagonism. For purposes of exposition, we shall talk primarily in terms of "on-center" units; it should be kept in mind that the same arguments can apply (with reversal in the excitation and inhibition) to "off-center" units. First, for both equal and unequal energy cases, inhibition should be expected to decrease in amplitude and perhaps change in rate with increase in distance between target and mask; thus, as the visual angle between target and mask is increased, the metacontrast effect ought to diminish and change shape. There is empirical evidence that the metacontrast effect does diminish and change shape: Alpern (1953) showed that with a center rectangle as target and two flanking rectangles as mask, as the visual angle between them increased to about $2 \mathrm{deg}$, the metacontrast effect diminished rapidly, and its maximum occurred at smaller and smaller values of $\Delta t$. Second, there ought to be fairly complicated changes in the metacontrast function with changes in the energy level of targets and masks, which are themselves of relatively equal energy, since there seem to be fairly complicated changes in excitation and inhibition with changes in the energy levels of stimuli. With increase in energy, a number of things may be expected to occur: excitation might be expected to decrease in gain and to develop a faster rate of response (Sperling \& Sondhi, 1968; Matin, 1968; Fuortes \& Hodgkin, 1964); inhibition might be expected also to decrease in gain and to increase in rate, although not necessarily in parallel with the changes in excitation; peripheral antagonism might be expected to contribute more and more to the center of the receptive field when the target is presented alone (Cleland \& Enroth-Cugell, 1968). At the same time, if there were not sensitivity and rate constant changes with increase in energy, the maxima of the metacontrast functions might be expected to occur at larger and larger values of $\Delta t$, due to the development of fuller inhibitory and excitatory responses, while the amplitude of the metacontrast function might decrease, due to the differentially higher level of excitation minus inhibition at maximum masking. This decrease in the amplitude of metacontrast might be expected to become smaller and smaller with increases in energy, due to the effects of reduced gain of excitation and inhibition. This presents a very tangled picture; we have discussed it in some detail because it is important to note that if the theory of metacontrast effects is based on rates of neural excitation and inhibition, then the ways in which these rates change must be carefully considered. What we would predict at this point, however, is simply, very generally, that metacontrast functions would vary a good deal with changes in energy. ${ }^{4}$ Thus, one aim of our experiment was 
concerned with replicating Kahneman's finding that increasing durations did not affect the metacontrast function; we would predict that it would.

Similarly, another aim of our study was to explore what would happen when the luminance of the stimuli was increased. As discussed above, we had no clear predictions on the behavior of metacontrast functions with changes in luminance, since there are a number of things that would be expected to happen simultaneously; but we did know that, empirically, good metacontrast functions have generally been obtained at relatively high energy levels $(1-2 \log \mathrm{ft}-\mathrm{L})$. Thus, if there are differences between apparent motion and metacontrast, these might be clearest where the obtained metacontrast effect is sharpest. Since the category rating scale that Kahneman used does not provide its own "anchor," and since the luminance variation in our study occurred in blocks, i.e., was not randomized from trial to trial, it is likely that this improvement in the metacontrast effect might not so much affect the amplitude of the metacontrast ratings as the shape of the functions, with better metacontrast functions describing more clearcut "U's." For apparent motion, we expected Korte's second, third, and fourth laws to hold. That is, we expected that with increase in luminance and duration, the maxima of the apparent motion functions would shift towards the origin; with increase in visual angle between stimuli, the maxima of the apparent motion functions should shift away from the origin. These laws would not contradict the assumption that motion analyzers mediate the apparent motion effect; however, with the model formulated at this qualitative a level, replication of these laws would not necessarily be taken as confirmation of the model. In addition, we expected that apparent motion would not diminish in amplitude nor change its overall shape with changes in visual angle between stimuli, changes in luminance, and changes in duration, on the assumption that if the effect is mediated by motion analyzers, then one would expect a range over which different analyzers would be maximally sensitive and a range of receptive field widths; thus, when one analyzer was not maximally stimulated, another one would be.

In summary, we expected that at least one of these three variables-separation between stimuli, luminance, and duration-and especially on the first (separation between stimuli), since the latter two may present a tangled picture, metacontrast and apparent motion functions would diverge.

Our study varied luminance, visual angle, and duration, and, in one experiment, viewing conditions (monoptic vs dichoptic). Our major hypothesis was that with increase in visual angle, metacontrast functions would diminish in amplitude and change shape; and that apparent motion functions would not diminish in amplitude and would change shape in the opposite direction. Our second hypothesis was that with changes in stimulus energy there would be a divergence of the metacontrast and apparent motion functions.

\section{GENERAL PROCEDURE}

The major variation across experiments was the luminance. For Experiment 1, the luminance was $1 \mathrm{ft}-\mathrm{L}$ (compared with Kahneman's $.925 \mathrm{ft}-\mathrm{L}$ ); Experiment 2, $2 \mathrm{ft}-\mathrm{L}$; Experiment 3, $6 \mathrm{ft}-\mathrm{L}$; and Experiment 4, $12 \mathrm{ft}-\mathrm{L}$. All luminances were measured by an S.E.I. photometer and were monitored daily by photocells placed in each of the channels whose output was displayed on a Tektronix Oscilloscope, Model 504.

The major variations within experiments were the visual angle and the duration of the stimuli. Measured from center of one square to center of an adjacent square, the visual angles were $55 \mathrm{~min}$ (identical to Kahneman's display), $2 \mathrm{deg}$, and 3 deg $20 \mathrm{~min}$. In Experiment 4 , in addition, a visual angle of $39 \mathrm{~min}$ was also used. This left about $.5 \mathrm{~min}$ of visual angle between adjacent sides of squares. These angles shall be called $\theta 0, \theta 1, \theta 2, \theta 3$, in order from smallest $(39 \mathrm{~min})$ to largest ( $3 \mathrm{deg} 20 \mathrm{~min}$ ). The stimuli were presented at each of three durations: 25,50 , and $125 \mathrm{msec}$. The duration of center and flank or flanks always equalled each other.

As much as possible, we attempted to replicate the Kahneman procedure. The following changes were made. For Experiments 1, 2, and 3, instead of a complete outline square, a square "C" was used. Instead of binocular viewing, Ss viewed the displays monoptically (and, in Experiment 4, dichoptically, in addition). Some. Ss served in more than one experiment; thus, after the first experiment, they could not be called naive. Ss' ratings were taken over a total of about $3 \mathrm{~h}$ (or $8 \mathrm{~h}$, in Experiment 4) as compared to the $2 \mathrm{~h}$ of Kahneman's Ss. Luminance levels varied from $1 \mathrm{ft}-\mathrm{L}$ (nearly identical to Kahneman's $.925 \mathrm{ft}-\mathrm{L}$ ) to more than $1 \log$ unit higher than in Kahneman's study. Stimuli were studied at three durations, as compared to the five durations (see B above) in Kahneman's study.

Our procedure, in detail, was this: Displays were presented in a six-channel binocular tachistoscope (Scientific Prototype M.ınufacturing Corporation, Type G). A fixation point was presented $5 \mathrm{~mm}$ or $1 \mathrm{deg} 09 \mathrm{~min}$ above a center square, as measured from the center of the square. A side of a square measured $2.7 \mathrm{~mm}$, subtending $37 \mathrm{~min}$ of visual angle at the $41-\mathrm{cm}$ viewing distance and 1.65 lens magnification of the tachistoscope. Stimuli were viewed monoptically, with the exception of Experiment 4, in which two conditions of viewing, monoptic and dichoptic, were used. In the monoptic conditions, the display was always presented to the S's right eye. The S's head was supported by an adjustable head and chin rest.

The instructions read to the $S$ are described in Kahneman (1967). The pertinent sections for metacontrast or apparent motion were repeated before each day's session. Questions were answered by repeating relevant sections of the instructions.

The $E$ began each stimulus presentation by saying, "Ready." He then initiated the display sequence. The fixation point appeared and remained on for the entire sequence. The center square was flashed $800 \mathrm{msec}$ after the fixation point appeared, followed at Interval $\Delta t$ either by both flanking squares for metacontrast or by one of the flanking squares for apparent motion. The interval between stimulus presentations was approximately $6 \mathrm{sec}$.

Each of the two major conditions, metacontrast and apparent motion, was studied with three sizes of visual angle (except for Experiment 4, in which four sizes of visual angle were used) in each of three durations of exposure for center and flank or flanks: 25,50 , and $125 \mathrm{msec}$, at each of 11 values of $\Delta t$, in each of four experiments, with different luminance levels per experiment.

For each experiment, the 11 values of $\Delta t$ for any one of the nine combinations of visual angle and exposure duration constituted one trial. Each trial was repeated four times in Experiments 1, 2, and 3, and five times in Experiment 4 . The $\Delta t$ 's within each trial were randomized as were the 36 (or 48 , in Experiment 4) trials for each of the two major conditions. For each $\mathrm{S}$, there were four 40-min sessions of 18 trials each, or, in Experiment 4, eight $60-\mathrm{min}$ sessions of 30 trials each.

The $\mathrm{S}$ was dark-adapted for $10 \mathrm{~min}$ prior to each day's session. Two practice trials preceded an initial encounter of a major condition. For each of the four practice trials, the visual angle was $\theta 1=55 \mathrm{~min}$ at an exposure duration of $50 \mathrm{msec}$.

The minor variations in procedure between experiments were as follows:

\section{Experiment 1}

Five undergraduates were paid to be Ss. Each was naive to 
this kind of experiment. A trial consisted of the 11 values of $\Delta t$, also used by Kahneman: $2,25,50,75, \ldots 250 \mathrm{msec}$. The order of presentation of the major conditions was counterbalanced for each $S$. The luminance at indefinite exposure was $1 \mathrm{ft}-\mathrm{L}$.

\section{Experiment 2}

Experiment 2 used four undergraduates who had served as Ss in a pilot study. A trial consisted of the same 11 values of $\Delta \mathrm{t}$ as in Experiment 1. Luminance at indefinite exposure was $2.0 \mathrm{ft}-\mathrm{L}$.

\section{Experiment 3}

Three of the five Ss who served in Experiment 1 also served in Experiment 3. A trial consisted of the same 11 values of $\Delta t$ as in Experiments 1 and 2. Luminance at indefinite exposure was $6.0 \mathrm{ft}-\mathrm{L}$.

\section{Experiment 4}

Three undergraduates served as Ss in Experiment 4. Each was naive to this kind of experiment. A trial consisted of 11 values of $\Delta \mathrm{t}$ which differed slightly from those used in the previous experiments: $0,20,50,70,120,150,175,200,250$, 300 . Four visual angles were used: the same three used in the other three experiments and an additional visual angle of $39 \mathrm{~min}(\theta 0)$. Dichoptic viewing conditions, in all other respects identical to monoptic viewing conditions, were also run. Each $\mathrm{S}$ now had a random order of presentation of major conditions for each type of viewing which was counterbalanced. Instead of an outline square C, a complete outline square was used. Luminance at indefinite exposure was $12 \mathrm{ft}-\mathrm{L}$. To find the proper setting of the tachistoscope for fusion to occur under dichoptic viewing, the entire stimulus display for metacontrast at $\theta 1=55 \mathrm{~min}$ was turned on for indefinite exposure. The $S$ then adjusted the viewing angle of the right field of the tachistoscope until the display appeared stabilized. In addition, two 90-deg prisms in each field could be adjusted so that the fields were superimposed. When the display was fused, a fixation point was turned on, and this was also fused.

\section{RESULTS}

Five five-way and one six-way analyses of variance were performed on the data. 5 Tables 1 and 2 summarize the significant effects. The error terms in all $F$ ratios were the next-order interaction involving Ss (McNemar, 1955). Table 1 describes five-way analysis effects in all experiments. Table 2 describes, in addition, the effects from a six-way analysis of variance performed on Experiment 4 . In each table, 1 refers to Ss, 2 to visual angle $(\theta), 3$ to duration of stimuli, 4 to major condition (apparent motion or metacontrast), and 5 to delay between presentation of center and flank or flanks (onset to onset, $\Delta \mathrm{t}$ ). In Table 2, 6 , in addition, refers to viewing condition (monoptic or dichoptic).

The metacontrast and apparent motion functions for the 25 -msec duration for the three values of $\theta$ in Experiments 1, 2, and 3 , and for the four values of $\theta$ in Experiment 4, for monoptic viewing, are shown in Fig. 1. Each point is the mean of each S's mean rating over Ss. Larger ratings indicate better effects: "good," apparent motion, and "effective," metacontrast. Each ordinate runs from 0 to 5 , as indicated by the general scale at the right; each abscissa runs from 0 to $250 \mathrm{msec} \Delta \mathrm{t}$ (except Experiment 4 which runs from 0 to $300 \mathrm{msec} \Delta t$ ) as indicated by the general scale in the middle of the figure. The metacontrast and apparent motion functions for $\theta 1$ for the three values of duration are shown in Fig. 2 . Ratings and scales are the same as in Fig. 1. Figure 3 shows the metacontrast and apparent motion functions for four values of $\theta$ for Experiment 4 for monoptic and dichoptic viewing for
Table 1

5-Way Analysis of Variance: Significant Effects

1: Subjects. 2: $\theta$. 3: Duration. 4: Major Condition. s: $\Delta t .(p<.05){ }^{*}=p<.01$.

\begin{tabular}{cccccc}
\hline Experiment & 1 & 2 & 3 & 4. Monoptic & 4. Dichoptic \\
\hline & & & & & 2 \\
3 & & 4 & & 4 \\
5 & 5 & 5 & $5^{*}$ & 5 \\
& & & 24 & $24^{*}$ & $23^{*}$ \\
& $25^{*}$ & 25 & 25 & $25^{*}$ & $25^{*}$ \\
34 & & 35 & $35^{*}$ & $35^{*}$ \\
& & & $45^{*}$ & & \\
& & & & 235 & \\
& & & $345^{*}$ & $245^{*}$ & $245^{*}$ \\
\hline
\end{tabular}

two values of duration (50 and $125 \mathrm{msec}$ ). Ratings and scales are the same as in Fig. 1.

There are two classes of effects which critically test Kahneman's hypothesis: first- and second-order interactions which involve the major condition. Differences between apparent movement and metacontrast will be expected in first-order interactions as (1) a difference in the shape of the functions for the two major conditions (Interaction 45), or (2) a difference in the amplitudes between the two conditions for different values of some variable (Interaction 4 ), or second-order interactions as variations in the shape of the function for the two major conditions for different values of some variable (Interaction 45 ).

The presence of a significant main effect for metacontrast vs apparent motion (4) is to be expected only if one or the other effect is rated consistently higher than the other, or if the shape of one varies over $\Delta t$ in such a way that its overall mean differs from that of the other. Since a number of widely varying shapes will give the same overall means, the presence of an insignificant effect here is neutral to the testing of Kahneman's hypothesis.

Our first major hypothesis was that apparent movement would remain constant in amplitude as visual angle increased, but would change shape, with maxima occurring at larger values of $\Delta \mathbf{t}$ (Korte's third law); and that metacontrast would diminish in amplitude as visual angle increased and would probably also change maxima in the opposite direction following Alpern (1953). Experiments 2, 3, and 4 resulted in significant differences in the behavior of the metacontrast and apparent motion functions with changes in visual angle; Experiment 1 did not show significant differences, although it is clear from Fig. 1 that the trend in Experiment 1 is in the right direction. For Experiment 1, on a Wilcoxon signed rank test, subtracting the mean for each $\Delta t$ at $\theta \mathrm{I}$ from the mean for each $\Delta t$ at $\theta 3$, and comparing the differences obtained on metacontrast vs those obtained on apparent motion, the differences are significant for $25 \mathrm{msec}$ at $\mathrm{p}<.025$, one-tailed, and for $50 \mathrm{msec}$ at $\mathrm{p}<.05$, one-tailed. In other words, the difference in amplitude between $\theta 1$ and $\theta 3$ on metacontrast is greater than the difference in amplitude between $\theta 1$ and $\theta 3$ on

Table 2

6-Way Analysis of Variance: Significant Effects

1: Subjects. 2: $\theta$. 3: Duration. 4: Major Condition. 5: $\Delta t$. 6: Viewing Condition. $(p<.05) .^{*}=p<.01$.

\begin{tabular}{cccc}
\hline Main & 1st Order & 2nd Order & 3rd Order \\
\hline $5^{*}$ & $24^{*}$ & $245^{*}$ & $2345^{*}$ \\
& $25^{*}$ & $246^{*}$ & \\
& 26 & $256^{*}$ & \\
& $35^{*}$ & & \\
\hline
\end{tabular}




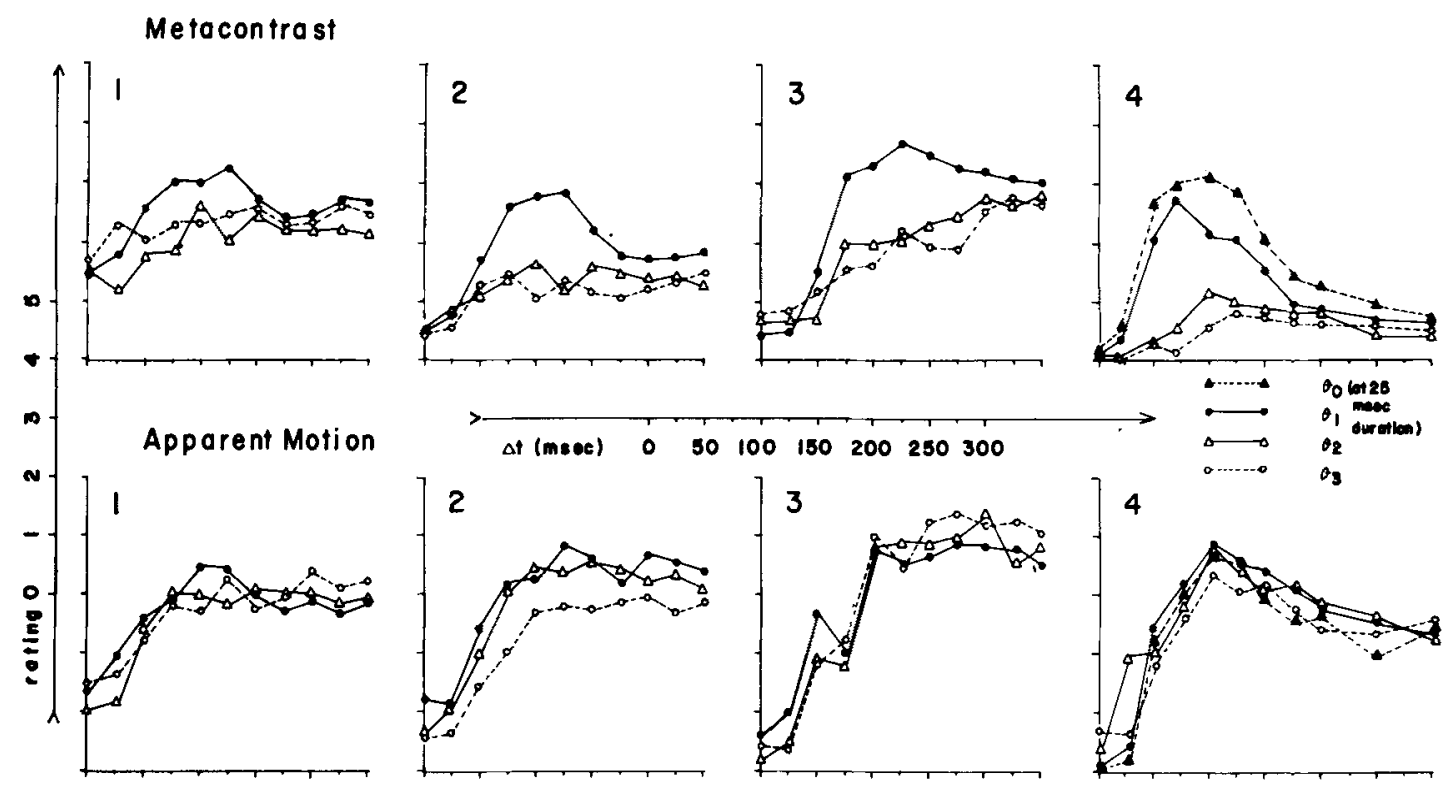

Fig. 1. The metacontrast and apparent motion functions for the $25-\mathrm{msec}$ duration for the three values of $\theta$ in Experiments 1, 2, and 3, and for the four values of $\theta$ in Experiment 4 for monoptic viewing.

apparent motion in Experiment 1 for the two lowest durations.

The hypothesis that metacontrast would diminish in amplitude with changes in visual angle while apparent motion would not was confirmed by Experiments 3 and 4 (Interaction 24) and can be seen from inspection of Figs. 1 and 3. In Experiment 3, for apparent motion there was a slight but consistent trend upwards with increase in visual angle; in Experiment 4 for apparent motion the order of amplitudes was $\theta 1, \theta 2, \theta 0$, and $\theta 3$ for monoptic viewing conditions, and $\theta 1, \theta 0, \theta 3, \theta 2$ for dichoptic viewing conditions. Figure 1 also shows some drop in amplitude with increasing $\theta$ for metacontrast as compared with apparent motion for Experiments 1 and 2, although this interaction did not reach significance on the analysis of variance. The Wilcoxon yielded significant visual angle effects for 25 and $50 \mathrm{msec}$, Experiment 1 , as noted above, and significant visual angle effects for $50 \mathrm{msec}$, Experiment $2(\mathrm{p}<.01)$. The hypothesis that the shape of metacontrast would differ from that of apparent motion as visual angle increased was confirmed for Experiments 2 and 4 (both monoptic and dichoptic), and can be seen also for Experiment 3, although here this interaction did not quite reach significance. The shape changes were, for the most part, contrary to predictions. The metacontrast functions did not so much shift their maxima to later $\Delta t^{\prime} s$ as they simply flattened out and lost the appearance of a "U." The apparent motion functions did not seem to shift their maxima either, with changes in visual angle; by and large, they simply remained at a constant, broad maximum over changes in visual angle. Thus, the experiments failed to confirm Korte's third law.

For large visual angles, then, it is clear that the apparent motion and metacontrast functions differ. For small visual angles also, however, the apparent motion and metacontrast functions differ. It can be seen by inspection of Fig. 1 that, in general, for small visual angles, metacontrast functions yield U-shapes. For these same visual angles, apparent motion functions yield either L-shapes or (for Experiment 4, $\mathrm{J}$-shapes). Since this description in effect only considers the large $\Delta t$ 's, this difference between the two conditions would not show up as a significant interaction on an overall analysis of variance. But, if, for the small visual angles, the drop in metacontrast functions over increasing values of $\Delta t$ is compared with the corresponding behavior of apparent motion functions over these same values of $\Delta t$ (for $\theta 0$ and $\theta 1$ for 25 , 50 , and $125 \mathrm{msec}$ for all experiments) then it is apparent that the shapes of the two functions do indeed differ. If each preceding point, beginning at $\Delta t=100 \mathrm{msec}$, is subtracted from each subsequent point, and a sign test performed on the signs of the differences, for metacontrast, the results are highly significant $(p<.001)$; for apparent motion, the results are insignificant. Thus, for small visual angles, for late $\Delta t$ 's, metacontrast is decreasing monotonic; apparent motion functions are not.

(For Experiments 1 and 3, the failure to obtain significant shape interactions in these two cases might be explained by the fact that under what could be assumed "best" conditions of visual angle, the metacontrast function displayed this drop to a much smaller extent.)

Our second hypothesis was that metacontrast functions would be extremely sensitive to changes in the energy level of the stimuli, whereas apparent motion functions might be expected to shift maxima with changes in duration and luminance (Korte's second and fourth laws) but otherwise remain relatively the same. Differences in the major conditions over luminance changes cannot be directly compared since different Ss were used, and since the type of rating scale employed provides no "anchor" or standard reference from one experiment to the next. But at each luminance, differences can be examined. In Experiment 1, where stimuli were at the lowest luminances used in this study, there was a significant decrease in amplitude for metacontrast with increase in duration, with no corresponding decrease in apparent motion amplitudes (Interaction 34, and Fig. 2). This reduction in amplitude was not accompanied by a flattening of the functions, as was the reduction in amplitude due to increases in visual angle. Indeed, as the duration increased, and, in general (with the exception of No. 3), across luminances, as the energy increased, the functions seemed to become more and more U-shaped. This is, however, simply a qualitative impression. In Experiment 3, there was a significant interaction between the shapes of the functions for apparent motion and metacontrast, and their durations (Interaction 345); this reflects the rise in apparent motion 


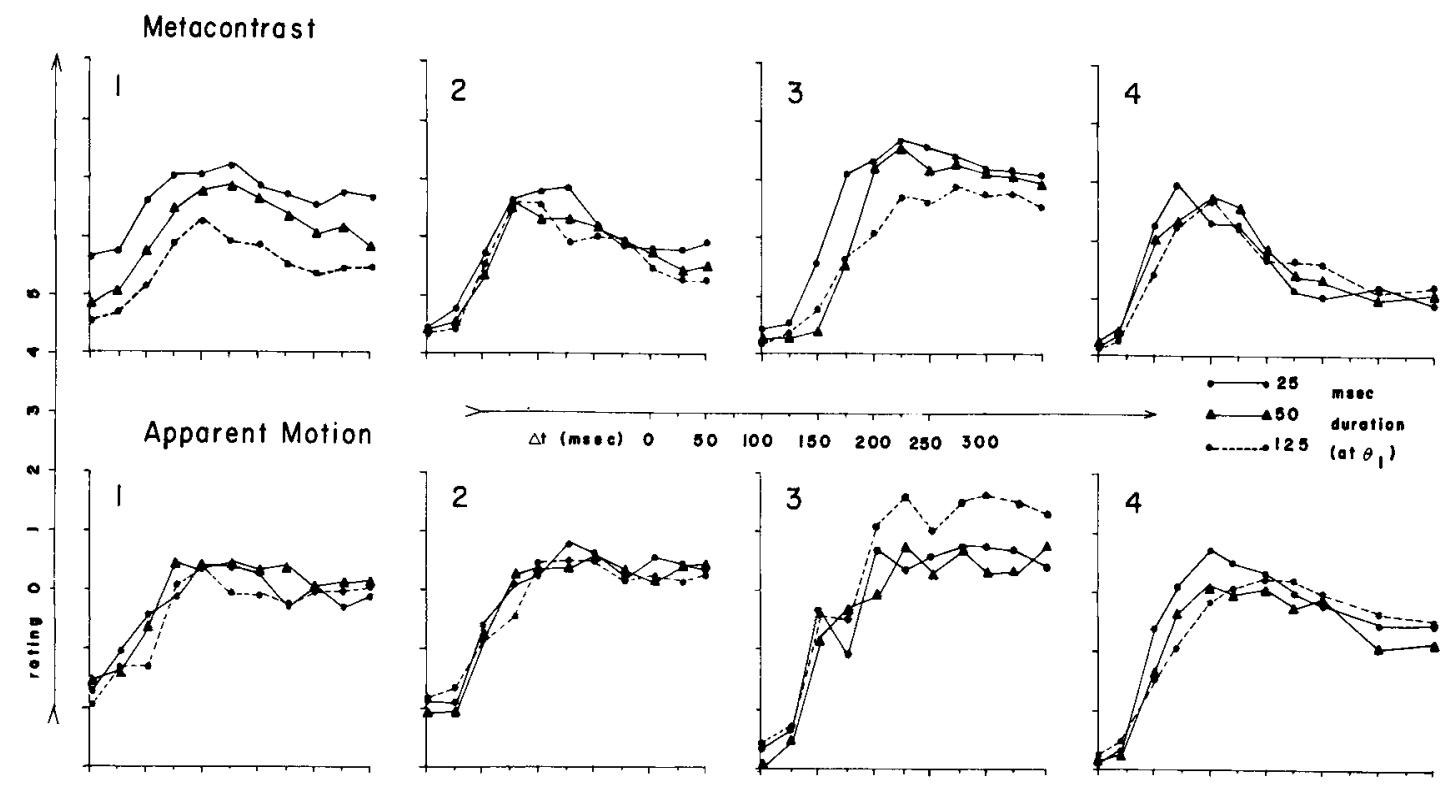

Fig. 2. The metacontrast and apparent motion functions for $\theta 1$ for the three values of duration for Experiments 1, 2, 3, and 4 for monoptic viewing.

amplitudes for large durations at late $\Delta$ t's as compared with a drop in metacontrast amplitudes for large durations at the same $\Delta t$ 's.

With the exception of Experiment 3 , it can be said that as luminance increased, and, for the lowest luminance, as duration increased, the metacontrast functions became more sharply defined, that is, the "U" became more pronounced. This sharpening of the metacontrast function then led to an increase in the number of significant effects, since the apparent motion function remained relatively constant. All experiments showed a significant interaction between visual angle and delay; hence, for both metacontrast and apparent motion, the functions for different visual angles are in no sense parallel. However, distinct changes in maxima for different visual angles, an expected result, was not confirmed. Experiments 3 and 4 , both monoptic and dichoptic conditions, also yielded a significant interaction between duration and delay; here, metacontrast and apparent motion combined did seem to have a shift in maximum at larger durations toward later $\Delta t$ 's. In Experiment 3, there was a significant difference in the shapes of the metacontrast and apparent motion functions (Interaction 45); this reflected the tendency for apparent motion to rise to a maximum and stay there across variations both in duration and visual angle, while the metacontrast functions at small visual angles described a "J," and at larger visual angles, simply increased monotonically with increases in $\Delta t$ (see Fig. 1). Both Experiments 3 and 4 (dichoptic) yielded a significant main effect between apparent motion, reflecting in Experiment 3 a larger overall mean amplitude for apparent motion which was a joint function of the "L" shape for apparent motion in Experiment 3, and of the higher overall mean ratings for apparent motion as compared to metacontrast. For Experiment 4, dichoptic, the significant main effect reflected the smaller overall mean amplitude for metacontrast as compared with apparent motion. This was a function of the marked drop in ratings for metacontrast as visual angle decreased; the overall mean for metacontrast was then much smaller than for apparent motion. Experiment 3 yielded some anamolous effects: the metacontrast functions were less sharp than was expected at $6 \mathrm{ft}-\mathrm{L}$, and the apparent motion functions differed in appearance from those of the other experiments. In addition, Experiment 3 was the only one in which the apparent motion function for $125-\mathrm{msec}$ duration was much higher than for the other durations. We are not sure how to explain these inconsistencies; however, it may be simply sample size. The least number of measurements were taken in Experiment 3; perhaps with additional measurements, the functions would have become less anamolous.

Experiment 4 deserves some individual discussion as it is the most elaborated of the experiments, using in addition to the three other values of visual angle, a visual angle of almost 0 deg between adjacent squares and presenting stimuli dichoptically as well as monoptically. The use of the additional visual angle merely confirmed the relationship already indicated in the other experiments: for the metacontrast function, the smallest visual angle yielded the greatest effect; for the apparent motion function, in general, the two medium visual angles yielded the greatest effects (see Fig. 3). This was as true for dichoptic viewing as for monoptic viewing (Table 2); in fact, the differences between visual angles and amplitudes of metacontrast for dichoptic viewing were more pronounced than those for monoptic viewing. When a six-way analysis of variance was performed on the data (Table 2), in addition to the retention of the significant effects mentioned above, additional effects appeared: the differences in the shape of metacontrast and apparent motion functions due to differences in visual angle varied additionally as a function of changes in duration (Interaction 2345); and monoptic and dichoptic viewing conditions led to variations between metacontrast and apparent motion as visual angle increased (Interaction 246). This former effect indicates that, whereas the effect of visual angle on duration is relatively constant for apparent motion (the higher the duration, the later the maximum, and a relatively constant difference between duration functions over the range of visual angles), for metacontrast, the order of duration maxima varies with changes in visual angle and the difference between duration functions vary from large differences at small visual angles to small differences at large visual angles. Notice that the effect for apparent motion reverses Korte's fourth law; there is a direct, rather than inverse, relationship between duration and $\Delta t$ at which optimal apparent motion is obtained.

The second effect, interaction between monoptic and dichoptic viewing conditions, visual angle, and apparent motion and metacontrast (Interaction 246), indicates that whereas at the very small visual angles, dichoptic metacontrast describes a "J-shape" with a high y-intercept, and monoptic 

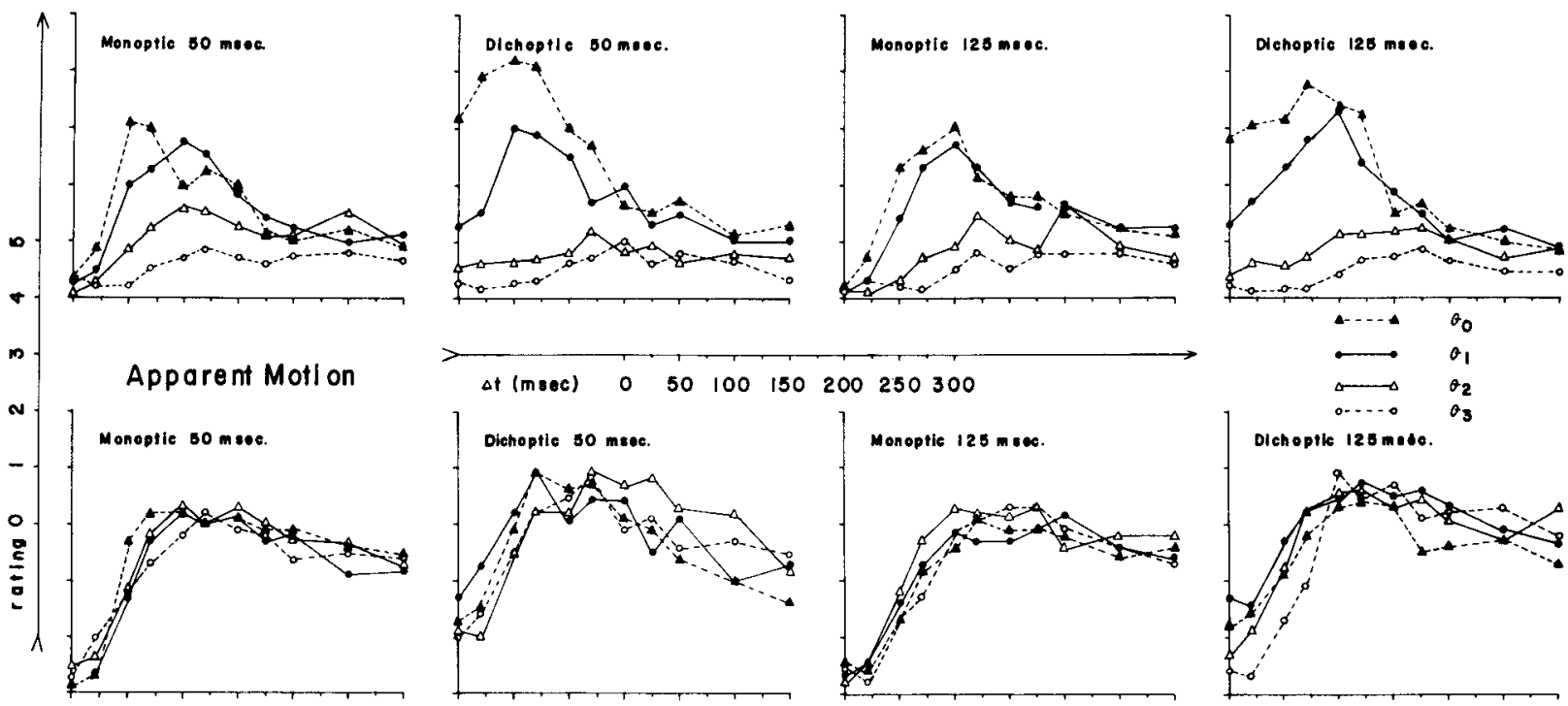

Fig. 3. The metacontrast and apparent motion functions for four values of $\theta$ for monoptic and dichoptic viewing for 50- and 125-msec duration in Experiment 4.

metacontrast does not, as visual angle increases, the $y$-intercept on dichoptic metacontrast functions moves towards zero. Apparent motion, on the other hand, changed very much less between dichoptic and monoptic viewing conditions because, whereas there was some raising of the $y$-intercept at small visual angles for dichoptic viewing, this was slight compared to the corresponding metacontrast function, and the drop to zero for the dichoptic functions for the large visual angles was therefore much smaller. In addition, the relative amplitude of dichoptic to monoptic apparent motion functions remained constant over visual angle, with dichoptic apparent motion rated about .25 points higher than monoptic apparent motion, while dichoptic metacontrast was rated about one point higher than monoptic functions at the small visual angles, a difference which steadily decreased to zero at the large visual angles.

It is clear from these data that metacontrast and apparent motion are following different laws. In all experiments, there were significant differences between apparent motion and metacontrast: at the lowest luminance, the difference was found primarily in the changing amplitude of the metacontrast effects and constant amplitude of apparent motion effects with differences in duration of the stimuli; at the higher luminances, along with some duration differences, metacontrast and apparent motion functions behaved in entirely different manners with increases in visual angle. At the small visual angles, the shapes of the two functions differ: metacontrast decreases monotonically at the late $\Delta t$ 's whereas apparent motion, in general, does not. In brief, metacontrast functions are much more sensitive to change, primarily to visual angle changes, and secondarily to duration, luminance, and viewing condition changes.

\section{DISCUSSION}

Metacontrast and apparent motion share in common the fact that under some conditions they both yield U-shapes which somewhat resemble each other. They cannot explain each other, however, because they are not one and the same phenomenon. Metacontrast decreases in amplitude and changes shape with increase in visual angle; apparent motion does not. This is the main difference found in the present study. There are a number of secondary differences. Metacontrast is sensitive to energy changes whereas apparent motion is not; metacontrast is sensitive to changes in viewing conditions whereas apparent motion is not; metacontrast exhibits a marked decline at the late $\Delta$ t's whereas apparent motion does not. Since Kahneman's hypothesis, and the apparent motion-metacontrast hypothesis, in general, requires an identity of behavior for the two psychophysical effects, the results of this study indicate that this hypothesis is untenable as an explanation of metacontrast.

The type of rating scale employed, and the lack of an anchor between one luminance level and the next, makes it difficult to establish precise quantitative descriptions of the behavior of the two types of effects. However, qualitative features of the two effects can be noted. We stated previously that we had in mind models both for apparent motion and metacontrast. A general lateral inhibition hypothesis for metacontrast assumes that the mask and target together fall on closely overlapping single unit receptive fields (most probably in the visual cortex) with the mask an inhibitory annulus or flank, and the target an excitatory center. (The target also inhibits the mask in this view, but due to the overlapping units, "edges" from the mask are still detected.) This kind of model would predict that as the distance between antagonistic stimuli increased, the inhibitory effects would diminish. Thus, a lateral inhibition model would be in no way inconsistent with the metacontrast effects obtained in this study. There is some evidence that with increase in energy, inhibition from the periphery of a receptive field is "turned on" and begins to influence the center of a receptive field in the absence of direct stimulation to the flanks (Cleland \& Enroth-Cugell, 1968); increases in inhibition of this type might play a role in the general increase (with the exception of Experiment 3 ) in "sharpness" of the metacontrast functions with increasing energy. In terms of maxima, there were no consistent trends towards or away from the origin with increases in energy, as might be predicted from rate constant and amplitude changes in excitation and inhibition functions; however, as noted, these changes would predict a tangled picture, with a number of effects possibly cancelling each other.

If metacontrast suggests an obvious neural analog which does not seem contradicted by the data found in this study, apparent motion suggests its neural analog too. These are the motion sensitive single units described by Barlow, Hill, and Levick (1964) and Barlow and Levick (1965) for the rabbit's retina. Although in man there is evidence that motion analysis has a central locus (DeValois, 1966), the mechanism of motion detection may be similar. This mechanism suggests a very 
simple model for apparent motion. An asymmetrical system of inhibition and excitation, with a single unit collecting from prior units which give transient initial bursts, and which are inhibited from cells to the left or right of themselves only, might fire only if it could sum stimulation from successive portions of a receptive field (and needs some offset in stimulus presentation in order that one of those portions is not inhibited). If human motion analyzers work on such a system, then the appearance of movement in apparent motion paradigms might be hypothesized to result from the stimulation of one of these units: the unit does not fire if only one short burst occurs, as might be the case for a stationary object; however, if two short bursts are summed, the motion detecting unit is excited above threshold and can fire. If the apparent motion effect is mediated by motion analyzers, then one would expect a range over which different analyzers would be maximally sensitive, and a range of receptive field widths; thus, with this model neither visual angle nor duration changes would be expected to affect the amplitude of the apparent motion functions, and, in general, in this study they did not. (Luminance changes would not be expected to affect the amplitude of the apparent motion functions either, but, as discussed, due to the lack of an anchor, the evidence for this prediction is equivocal.) The inhibition offsets and excitation rate constants would vary for units sensitive to different velocities; such differences in rates would be expected to produce differences in $\Delta \mathrm{t}$ at which the maxima of the apparent motions are found. Hence, it is not clear why, according to this hypothesis, we found very little apparent shifts in maxima with visual angle changes. This bears further investigation. Notice that inhibition in a motion analyzer plays the role opposite to what it is assumed to do in metacontrast: if there is apparent motion, or motion detection at all, this is because inhibition has not been activated, or has been activated in a direction which is opposite to the preferred direction of the unit. Thus, for these single unit analogies to apparent motion and metacontrast, the appearance of motion implies a lack of inhibition, the appearance of metacontrast, the presence of inhibition.

\section{CONCLUSION}

Kahneman (1967) and others have hypothesized that metacontrast is a case of "impossible" apparent motion. He has supported this hypothesis by a study which shows that, for five durations of target and mask at a luminance of $1 \mathrm{~mL}$ $(.925 \mathrm{ft}-\mathrm{L})$, the apparent motion functions resemble the metacontrast functions. The results of this present study, however, indicate that the resemblance between the two conditions is superficial. First, we failed to replicate the identity of metacontrast functions over three out of the five durations which Kahneman used, at a luminance level ( $1 \mathrm{ft}-\mathrm{L}$ ) very similar to the one he used. Second, at higher luminances, visual angle became of crucial importance: at small visual angles, typical metacontrast functions were obtained; at large visual angles, it was not clear that metacontrast was what was being obtained. Apparent motion, however, remained relatively constant in amplitude and, with some exception, in shape over increases in visual angle. Thus, the hypothesis that metacontrast is a special case of apparent motion does not seem tenable.

The extent to which these two psychophysical effects have a common basis, however, can be considered in a more general sense: both may have something to do with the neural circuitry involved in initial pattern analysis. Thus, a model for single unit motion analyzers suggests a mechanism whereby apparent movement would result; a model for simple cortical units with annular or flanking receptive field inhibition suggests a mechanism whereby metacontrast and paracontrast might be obtained. Neural models of single units, then, seem suggestive in explaining both effects; and it is in this sense, if any, that metacontrast and apparent motion may be related.

\section{REFERENCES}

ALPERN, M. Metacontrast. Journal of the Optical Society of America, $1953,43,648-657$.

BARLOW, H. B., \& LEVICK, W. R. The mechanisms of directionally selective units in the rabbit's retina. Journal of Physiology, 1965, 178, 477-504.

BARLOW, H. B., HILL, R. M., \& LEVICK, W. R. Retinal ganglion cells responding selectively to direction and speed of image motion in the rabbit. Journal of Phy siology, 1964, 173, 377-407.

BOX, G. E. P. Non-normality and tests on variances. Biometrika, 1953, $40,318-335$.

CLELAND, B. G., \& ENROTH-CUGELL, C. Quantitative aspects of sensitivity and summation in the cat retina. Journal of Physiology, $1968,198,17-38$

DEMBER, W. N., \& PURCELL, D. G. Recovery of masked visual targets by inhibition of the masking stimulus. Science, 1967, 157, 1335-1336.

DeVALOIS, R. L. Neural processing of visual information. In R. W. Russell (Ed.), Frontiers in physiological psychology. New York: Academic Press, 1966.

ERIKSEN, C., COLLINS, J. F., \& GREENSPON, T. S. An analysis of certain factors responsible for nonmonotonic backward masking functions. Journal of Experimental Psychology, 1967, 75, 500-507.

FEHRER, E. Effect of stimulus on retroactive masking. Journal of Experimental Psychology, 1966, 71, 612-615.

FUORTES, M. G. F., \& HODGKIN, A. L. Changes in time scale and sensitivity in the ommatidia of Limulus. Journal of Physiology, 1964, $172,239-263$

KAHNEMAN, D. An onset-onset law for one case of apparent motion and metacontrast. Perception \& Psychophysics, 1967, 2, 577-584.

KAHNEMAN, D. Method findings, and theory in studies of visual masking. Psychological Bulletin, in press.

LANDAHL, H. D. Neural model for interval discrimination. Unpublished manuscript, 1968.

McNEMAR, Q. Psychological statistics (2nd ed.). New York: John Wiley, 1955 .

MATIN, L. Critical duration, the differential luminance threshold, critical flicker frequency, and visual adaptation: A theoretical treatment. Journal of the Optical Society of America, 1968, 58, 404-414.

RATLIFF, F. Mach bands. Quantitative studies on neural networds in the retina. San Francisco: Holden Day, 1965.

SCHILLER, P. Single unit analysis of backward visual masking and metacontrast in the cat lateral geniculate nucleus. Vision Research, $1968,8,855-866$.

SCHILLER, P., \& SMITH, M. Detection in metacontrast. Journal of Experimental Psy chology, 1966, 71, 32-46.

SPERLING, G., \& SONDHI, M. M. Model for visual luminance discrimination and flicker detection. Journal of the Optical Society of America, 1968, 58, 1133-1145.

STEVENS, S. S. Duration, luminance, and the brightness exponent. Perception \& Psychophysics, 1966, 1, 96-100.

WEISSTEIN, N. A Rashevesky-Landahl neural net: Simulation of metacontrast. Psychological Review, 1968, 75, 494-521.

WEISSTEIN, N. What the frog's eye tells the human brain: Single cell analyzers in the human visual system. Psychological Bulletin, in press.

\section{NOTES}

1. This work was supported in part by a grant to the first author from the National Institutes of Neurological Diseases and Blindness (NB07461).

2. Address: Department of Psychology, Loyola University, 6525 North Sheridan Road, Chicago, Illinois 60626.

3. In a personal communication, Kahneman has noted that both apparent and real motion might be handled by a neural network similar to that described by Barlow et al (1964) and Barlow and Levick (1965) for the rabbit retina. This suggestion has been taken up in the model of apparent motion used in this paper.

4. Amplitude decreases and increases in $\Delta t$ at which maxima occur with increase in energy are predicted by the Weisstein (1968) model; changes in rate constant and sensitivity are not provided for by the model as it stands, although the incorporation of a luminance adaptation model such as that proposed by Sperling \& Sondhi (1968) would in no way contradict the components of the Weisstein (1968) model, but would simply constitute, in effect, an addition of neurons peripheral to those dealt with in the Weisstein model. However, with this addition, simulated metacontrast functions would change from what they were in the 1968 version.

5. We assume, as discussed by Stevens (1966), that the population distribution of ratings in this experiment is not normal; however, since it seems plausible that the departure from normality should be relatively the same across conditions, the analysis of variance test, being insensitive to nonnormality if the departure is consistent across groups, would seem an appropriate test to use (Box, 1953).

(Accepted for publication Jantary 6, 1969.) 